\title{
Evaluating the role of wheat histone variant genes in development and response to abiotic stress in Arabidopsis
}

\author{
H. LV ${ }^{1}$, X. CUI ${ }^{1}$, P. ZHANG ${ }^{1}$, Y. LI ${ }^{1}$, Y. JI ${ }^{2}$, Y. WANG ${ }^{3}$, G. XIA ${ }^{1}$, and M. WANG ${ }^{1 *}$ \\ The Key Laboratory of Plant Cell Engineering and Germplasm Innovation, Ministry of Education, School of \\ Life Science, Shandong University, Shandong 250100, P.R. China ${ }^{1}$ \\ College of veterinary medicine, Nanjing Agricultural University, Nanjing 210095, P.R. China ${ }^{2}$ \\ Shijiazhuang Academy of Agriculture and Forestry Sciences, Shijiazhuang 050041, P.R. China ${ }^{3}$
}

\begin{abstract}
Histone variants can epigenetically regulate gene transcription through chromatin modulation. This regulation have been occasionally found in responses to abiotic stresses in plants, but their roles are not quite clear. Here, we describe 12 saltresponsive histone variant genes isolated from wheat. There was no sequence polymorphism in these 12 genes between the wheat cultivar 'JN177' and its salinity and drought tolerant derivative 'SR3' indicating that histone variant genes are highly conserved. However, these genes displayed differential patterns of transcription in 'JN177' and 'SR3'. When transformed into Arabidopsis thaliana, eight of the genes were silenced. The heterologous expression of the four active transgenes had no discernible effect on the Arabidopsis phenotype neither under control conditions nor under different abiotic stresses suggesting that histone variants could not be considered as candidate genes for molecular breeding by ectopic expression.
\end{abstract}

Additional key words: chromatin remodeling, transcription regulation, transgenic plants, Triticum aestivum.

\section{Introduction}

Histone variants influence gene expression by modulating chromatin (Talbert and Henikoff 2010). Unlike the major histones, histone variants are constitutively transcribed, and can vary from the canonical forms with respect to both their primary amino acid sequence and post-translational modifications (Talbert and Henikoff 2010). In plants, histone genes have been associated with the response to abiotic stresses, but their precise role is still not well known. The tomato H1-S variant is drought-inducible, and its knockdown by antisense technology promotes stomatal closure and enhances drought tolerance (Scippa et al. 2004). The H2A.Z variant of Arabidopsis thaliana is involved in the response to phosphate starvation (Smith et al. 2010) and also in the correct perception of ambient temperature (Kumar and Wigge 2010). The role of these two histone variants in the adaptation to abiotic stresses was confirmed by knockdown/knockout of histone variants and/or their chromatin-depositing chaperones (Kumar and Wigge 2010, Scippa et al. 2004, Smith et al. 2010). Notably, it would be valuable if the overexpression of a gene can affect target phenotype(s) or agricultural trait(s) in different plants and/or crops because this determines the application potential of the gene in molecular breeding. However, whether the overexpression of histone variants of a plant species (such as wheat) affects the response to abiotic stresses in other plant species (such as $A$. thaliana) has not been investigated so far. Moreover, the amounts of variants are different among major histones, of which $\mathrm{H} 2 \mathrm{~A}$ variants are mostly diverse, $\mathrm{H} 3$ variants are not frequent, and $\mathrm{H} 2 \mathrm{~B}$ variants are rare (Kamakaka and Biggins 2005). Then, besides H1-S and H2A.Z, the involvement of other histone variants in the response to abiotic stresses needs to be unravelled.

Bread wheat is one of the most important food crops. We previously produced a wheat cultivar 'SR3' by a somatic hybridization between wheat cultivar 'JN177' and tall wheatgrass (Thinopyrum ponticum); 'SR3' has shown remarkable salinity and drought tolerance (Xia et al. 2003, Xia 2009). The comparison with its wheat progenitor has shown that numerous DNA sequence and epigenetic alterations are induced during its development, which lead to both transcriptomic and proteomic alterations

Submitted 13 September 2018, last revision 26 December 2018, accepted 2 January 2019.

Abbreviations: ABA - abscisic acid; H - histone; pI - isoelectric point; cDNA - complementary DNA. q - quantitative, RT - reverse transcription, sq - semiquantitative.

Acknowledgements: This research was supported by the National Transgenic Project (2016ZX08002002-003), the Natural Science Foundation of China (31570258), and the National Key Research and Development Project (2016YFD0102003).

* Corresponding author; fax: (+86) 531 88364525, e-mail: wangmc@sdu.edu.cn 
(Wang et al. 2008, Peng et al. 2009, Liu et al. 2012). Transcriptomic analysis has shown that a number of probes which are homologous to histone variants respond differentially to salinity stress, some of which appear differential responsive patterns between 'SR3' and 'JN177' (Liu et al. 2012). This suggests that histone variants are important in plant defence against this stress. If so, some of these genes could represent attractive targets for plant improvement. We previously established a strategy to confirm the function of wheat genes in A. thaliana (Shan et al. 2008, Gao et al. 2010, Li et al. 2010, He et al. 2012, Dong et al. 2013, Liu et al. 2014, Zhao et al. 2014). The aim of the present research was to isolate 12 histone variant genes and to determine the phenotypic consequences of their expression in A. thaliana.

\section{Materials and methods}

Growing conditions and salinity treatment: Wheat (Triticum aestivum L.) seedlings were grown to the threeleaf stage in a half-strength Hoagland solution under a 16-h photoperiod, an irradiance of $600 \mu \mathrm{mol} \mathrm{m} \mathrm{m}^{-2} \mathrm{~s}^{-1}$, day/night temperatures of $22 / 20{ }^{\circ} \mathrm{C}$, and a $60 \%$ relative humidity, and then transferred to the half-strength Hoagland solution either with or without $200 \mathrm{mM} \mathrm{NaCl}$ for $0-24 \mathrm{~h}$. Both leaves and roots were sampled for RNA isolation at a set time of the day.

Cloning and sequence analysis: The sequences of the differentially transcribed probes with an annotation as histone variants (Fig. 1 Suppl.) were subjected to BLASTn analysis against a wheat EST database hosted by $N C B I$, and all matching ESTs were assembled into a contig using the CAP3 package (Huang and Madan 1999). The contig sequence was used to guide the design of genespecific primer pairs targeting the open reading frame (ORF) of each gene (Table 1 Suppl.). The ORFs and the associated genomic sequences were amplified from the complementary DNA (cDNA) and genomic DNA of both 'SR3' and 'JN177'. Phylogenetic analyses of the predicted peptide sequences relied on the neighbour-joining method implemented in both CLUSTALX and MEGA3 softwares. Secondary structure and nucleus localization sequences (NLS) were predicted using PSIPRED (http://bioinf.cs.ucl. ac.uk/psipred/) and WoLF PSORT (http://wolfpsort.org/).

Quantitative (q) and semiquantitative (sq) reverse transcription (RT) PCR: Total RNA was extracted with a TRIzol reagent (Takara, Dalian, China) and then treated with RNase-free DNase (Promega, Madison, USA). The first strand cDNA was synthesized using an $M-M L V$ kit (Invitrogen, Carlsbad, USA). The cDNA was used as a template for RT-qPCR in $20 \mathrm{~mm}^{3}$ of solution containing $10 \mathrm{~mm}^{3}$ of $2 \times$ SYBR Premix Ex Taq mix (Takara), $0.2 \mu \mathrm{M}$ forward and $0.2 \mu \mathrm{M}$ reverse primers, $1 \mathrm{~mm}^{3}$ of a $1: 10$ dilution of the cDNA; the cycling regime comprised a denaturation step of $95^{\circ} \mathrm{C}$ for $2 \mathrm{~min}$ followed by 45 cycles of $95^{\circ} \mathrm{C}$ for $10 \mathrm{~s}, 60^{\circ} \mathrm{C}$ for $20 \mathrm{~s}$, and $72^{\circ} \mathrm{C}$ for $20 \mathrm{~s}$. A melting curve analysis was performed over the range of 80 to
$95{ }^{\circ} \mathrm{C}$ at $0.5{ }^{\circ} \mathrm{C}$ intervals. Relative gene expressions were detected using the $2^{-\Delta \Delta \mathrm{CT}}$ method (Livak and Schmittgen 2001). The RT-sqPCR cycling regime comprised an initial denaturation at $95{ }^{\circ} \mathrm{C}$ for 5 min followed by 28 cycles of $94{ }^{\circ} \mathrm{C}$ for $30 \mathrm{~s}, 55^{\circ} \mathrm{C}$ for $30 \mathrm{~s}$, and $72{ }^{\circ} \mathrm{C}$ for $45 \mathrm{~s}$ with a final extension at $72{ }^{\circ} \mathrm{C}$ for $5 \mathrm{~min}$. Wheat the $A C T I N$ gene (AB181991) or A. TUBULIN gene (AT1G04820) was used as an internal reference. All experiments were replicated at least three times using independent cDNA preparations with four technical replicates per biological replicate.

Subcellular localization assay: Histone ORFs lacking their termination codon were introduced into the pBI221 vector creating an in-frame histone/green fluorescent protein (GFP) fusion transgene under the control of the CaMV $35 \mathrm{~S}$ promoter. The fusion or an empty vector was transiently transformed into onion epidermis cells according to the protocol of Von Arnim (2007). After incubation at $21-23^{\circ} \mathrm{C}$ for $16 \mathrm{~h}$, a GFP signal was detected by confocal fluorescence microscopy (460 $\mathrm{nm}$ excitation, $535 \mathrm{~nm}$ emission). The assay of each protein was repeated twice. In each assay, at least 10 cells producing fluorescence were detected. The assay of each protein was repeated twice, and at least 10 cells producing GFP fusion protein were observed.

Generation of heterologous expression of Arabidipsis thaliana lines: The ORF of each histone gene was cloned into the pSTART vector driven by the CaMV 35 S promoter, and the constructs transformed into $A$. thaliana L. genotype Col-0 using the floral dipping method (Clough and Bent 1998). Homozygous transgenic lines were selected by kanamycin screening and confirmed by genomic PCR with A. thaliana DNA as a template. The transcription of the transgenes in $A$. thaliana was confirmed by RT-sqPCR as mentioned above. The primers are listed in Table 1 Suppl.

Analysis of the phenotypic effect on development: $A$. thaliana seeds were surface-sterilized by a $5 \mathrm{~min}$ immersion in $70 \%(\mathrm{v} / \mathrm{v})$ ethanol followed by a $10 \mathrm{~min}$ treatment with $5 \%(\mathrm{~m} / \mathrm{v}) \mathrm{NaOCl}$, and finally by several rinses in sterile water. The seeds were imbibed in the dark at $22{ }^{\circ} \mathrm{C}$ for $72 \mathrm{~h}$ by placing them on solidified a half strength Murashige and Skoog (1/2 MS) medium. Uniformly germinating seeds were transferred to a fresh plate, which was oriented vertically and held under a $16-\mathrm{h}$ photoperiod, an irradiance of $600 \mu \mathrm{mol} \mathrm{m} \mathrm{m}^{-2} \mathrm{~s}^{-1}$, day/night temperatures of $22 / 20{ }^{\circ} \mathrm{C}$, and a $60 \%$ relative humidity for 7 or $13 \mathrm{~d}$ before the length of the primary root and the lateral root density were measured. Seven-d-old seedlings were transferred into soil and grown under the same regime for two weeks to allow the measurement of the area of the sixth rosette leaf. Once the plants had reached the stage when the siliques had become partially desiccated, measurements of plant height, rosette leaf branch number, and cauline leaf branch number were made. Sets of soilplanted seedlings were held under either a $16-\mathrm{h}$ or an 8 -h photoperiod to measure the time taken to reach flowering. The mass of 1000 seeds and the seed yield per plant were also measured. 
Responses to abiotic stresses: Surface-sterilized A. thaliana seeds were germinated as mentioned above. Uniformly germinated seeds were transferred onto $1 / 2 \mathrm{MS}$ plates containing one of 50,100 , or $125 \mathrm{mM} \mathrm{NaCl} ; 100,200$, or $300 \mathrm{mM}$ mannitol; 1,3 , or $5 \mathrm{mM} \mathrm{H}_{2} \mathrm{O}_{2} ; 2,5$, or $10 \mu \mathrm{M}$ abscisic acid (ABA); 2, 5 or $10 \mu \mathrm{M}$ naphthalene acetic acid (NAA); 2, 5, or $10 \mu \mathrm{M}$ 1-aminocyclopropane-1-carboxylic acid (ACC). Then the plates were placed vertically under conditions mentioned above for $10 \mathrm{~d}(\mathrm{NaCl})$ or two weeks (other treatments). To assess drought tolerance, a waterwithholding assay was conducted. Surface-sterilized seeds of $A$. thaliana were plated on a $1 / 2 \mathrm{MS}$ medium. The plates were initially held at $4{ }^{\circ} \mathrm{C}$ in the dark for four days to break seed dormancy and then transferred to conditions mentioned above for $12 \mathrm{~d}$. The seedlings were transferred into moistened substrate containing soil and Vermiculite $(2: 1, v / v)$ for three weeks, after which water was withheld for a further three weeks before re-watering for three days. To assess salt tolerance, one-month-old seedlings planted in soil were irrigated with $200 \mathrm{mM} \mathrm{NaCl}$ every three days until the seedlings were wilted and dead, and the growth phenotype was observed during the treatment course.

Germination assay: Surface-sterilized $A$. thaliana seeds were laid on a solidified $1 / 2 \mathrm{MS}$ medium containing 50, 100 , or $125 \mathrm{mM} \mathrm{NaCl} ; 100,200$, or $300 \mathrm{mM}$ mannitol; 2 or $4 \mu \mathrm{M} \mathrm{ABA} ; 1,3$, or $5 \mathrm{mM} \mathrm{H}_{2} \mathrm{O}_{2}$ and held at $4{ }^{\circ} \mathrm{C}$ for $2 \mathrm{~d}$ in the dark before being transferred to the conditions mentioned above. A germinated seed was defined as the one which successfully opened its cotyledons.

Statistics: The difference between two samples was calculated using the Student $t$-test $(\alpha=0.05)$. The difference among more than two samples was calculated using the one-way ANOVA lowest significance difference (LSD) test $(\alpha=0.05)$.

\section{Results}

The genes of H1, H2A, H3, and $\mathrm{H} 4$ variants, which were among those previously identified as being salinity responsive (Liu et al. 2012), chosen for full-length cDNA isolation are listed in Fig. 1 Suppl. The RT-qPCR analysis of five genes ( $\mathrm{TaH} 2 \mathrm{~A}-2$, TaH2A-3, TaH2A-4, TaH3-2, and $\mathrm{TaH} 2 \mathrm{~A} . \mathrm{Z}$ ) revealed a diversity of responses to the salinity stress (Fig. 1). $\mathrm{TaH} 2 \mathrm{~A}-2$ was induced by $\mathrm{NaCl}$ treatment in leaves with a stronger induction in 'SR3' than in 'JN177' (Fig. 1A); it was strongly down-regulated by the $\mathrm{NaCl}$ stress in roots (Fig. $1 B$ ). TaH2A-3 was not responsive to $\mathrm{NaCl}$ treatment in leaves but was reduced gradually in roots under the $\mathrm{NaCl}$ stress, and the gene had a comparable transcript abundance in either leaves or roots of 'SR3' and 'JN177' at each detected time point (Fig. 1C,D). TaH2A-4 had a constant expression profile in leaves but was slightly induced under the $\mathrm{NaCl}$ stress in roots; its expression in leaves was comparable between the two cultivars, but in roots, TaH $2 A-4$ had a remarkably higher expression in 'SR3' than in 'JN177' (Fig. 1E,F). TaH3-2 was induced by the $\mathrm{NaCl}$ stress in both leaves and roots, and the expression was higher in 'SR3' than in 'JN177' (Fig. 1G,H). Given that H2A.Z is a widely studied histone variant and has proved to play a vital role in abiotic stresses, we isolated a wheat H2A.Z gene TaH2A.Z-1. TaH2A.Z-1 was induced in leaves but restricted in roots as the $\mathrm{NaCl}$ treatment was prolonged (Fig. 1I,J).

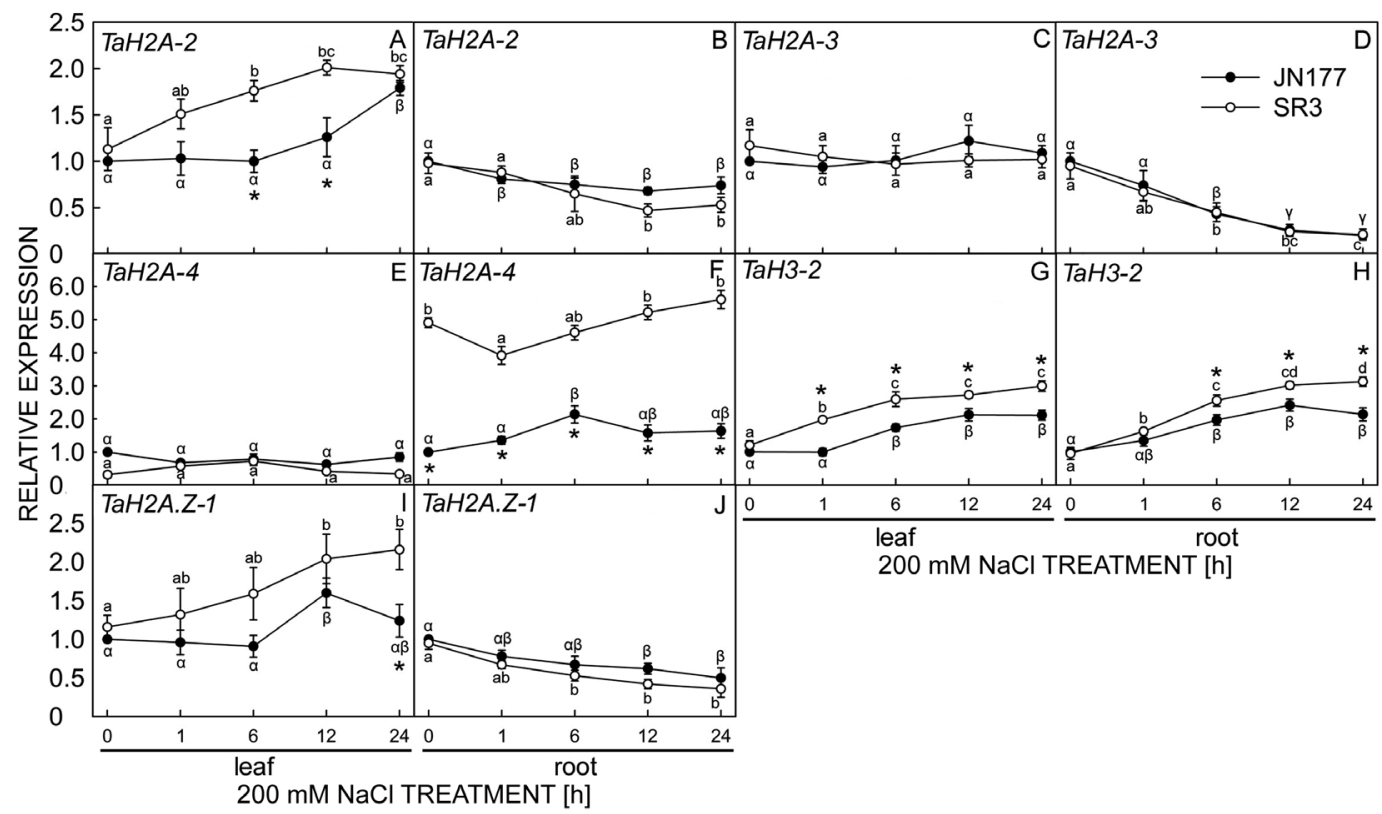

Fig. 1. The relative expression of wheat histone variant genes after exposure of a three-leaf stage wheat seedling to $200 \mathrm{mM} \mathrm{NaCl}$ as measure by reverse transcription quantitative PCR. Means \pm SDs, $n=4$. Differences among time points in either 'SR3' or 'JN177' were calculated with one-way ANOVA and those with different English or Greek letters are statistically significant $(P<0.05)$. The differences between 'SR3' and 'JN177' at each time point were calculated with the $t$-test, and those labelled with * are statistically significant $(P<0.05)$. 


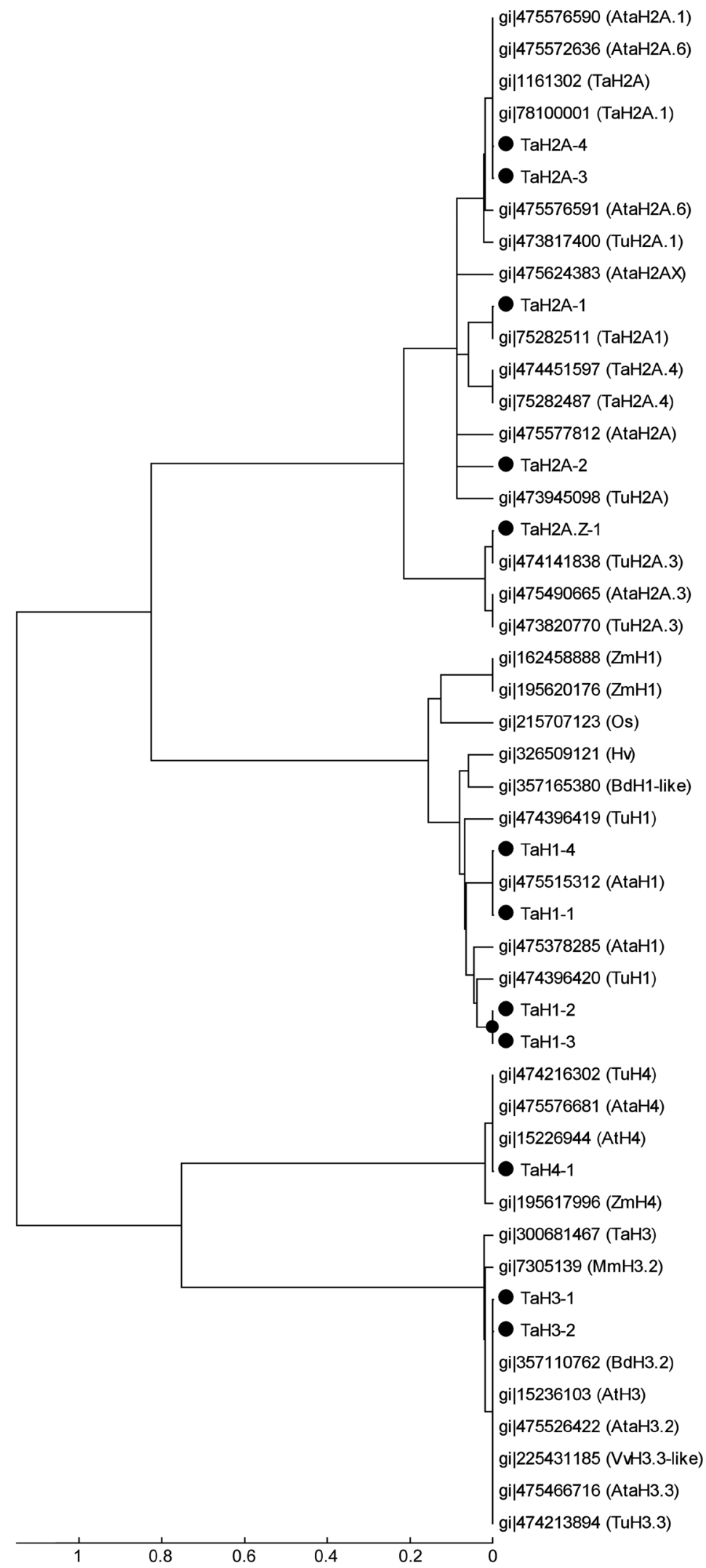

Fig. 2. Phylogenetic analysis of histone variant proteins. At - A. thaliana; Ata - Aegilops tauschii; Bd - Brachypodium distachyon; Hv - Hordeum vulgare; Mm - Mus musculus;Os - Oryza sativa; Ta - Triticum aestivum; Tu - Triticum urartu; Vv - Vitis vinifera; Zm Zea mays. 
Of the 12 histone variant genes cloned from 'SR3' and 'JN177', the ORFs of the $4 \mathrm{HI}$ variant genes TaH1-1 to TaH1-4 were amplified by the same primer pair although the amplicon lengths differed (Table 2 Suppl). Unlike major histone genes, some of histone variant genes have introns (Talbert and Henikoff 2010). Here, TaH1-1 to -4, TaH2A-4, and TaH3-2 all had an intron (Table 2 Suppl., Fig. 2 Suppl.). Although the 'SR3' genome differs from the 'JN177' genome by genome-scale genetic variation during asymmetric somatic hybridization (Wang et al. 2015, Xia 2009), no sequence variation was found in the ORFs of any of the 12 genes (Table 2 Suppl.).

The phylogenetic analysis of the gene predicted polypeptides shows that the $\mathrm{H} 1 / \mathrm{H} 2 \mathrm{~A}$ and $\mathrm{H} 3 / \mathrm{H} 4$ sequences formed two distinct clades (Fig. 2). TaH2A-3 and TaH2A-4 were almost identical to TaH2A.1 and TaH2A.6, and to their homologs in both Triticum urartu (an $A$ genome progenitor) and Aegilops tauschii (a $D$ genome progenitor). TaH2A-2 clustered the $\mathrm{H} 2 \mathrm{~A}$ proteins of T. urartu, Ae. tauschii, and barley. TaH2A-1 was distinct from the TaH2A-2, -3 , or -4 sequences, and it clustered instead with the H2A-7 homologs in bread wheat, T. urartu, and Ae. tauschii (but not with any of the H2A-7 homologs present in dicotyledonous species). TaH2A.Z-1 formed a recognizable sub-clade separated from the other $\mathrm{H} 2 \mathrm{As}$. In the H1 clade, TaH1-1 to -4 clustered with their homologs from T. urartu and Ae. tauschii, though not with homologs from other monocotyledonous species. In the H3 clade, TaH3-1 and TaH3-2 belonged to two different sub-clades, one homologous to H3 and the other to H3.2.

Histones are typically basic proteins rich in lysine and arginine. The $\mathrm{H} 1$ variants had a much higher lysine content than H2A, H3, or H4 (Table 3 Suppl.), but had the lowest ariginine content $(\sim 3 \%)$. The proteins (particularly the $\mathrm{H} 1 \mathrm{~s}$ ) also contained a high alanine content. The predicted pI of all of the products was $>10$ with the H3 and H4 proteins showing a $\mathrm{pI}$ of $>11$ (Table 3 Suppl.). The proteins included $3-5 \alpha$-helices and one nucleus localization sequence, whereas the $\mathrm{H} 1$ and $\mathrm{H} 2 \mathrm{~A}$ proteins in addition

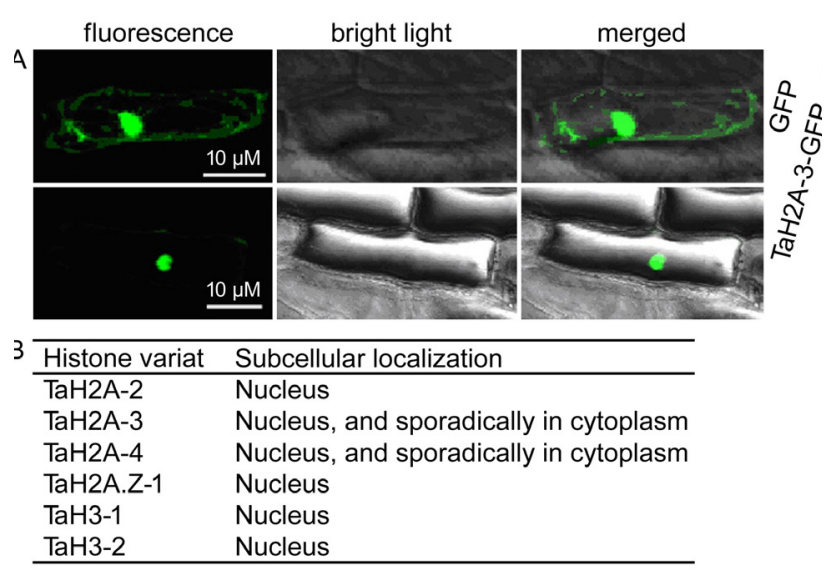

Fig. 3. Histone variants localize primarily in the nucleus. $A$ - Transient transcription of the TaH2A-3-GFP fusion transgene in onion epidermis cells. GFP - green fluorescent protein. $B$ - A summary of the sub-cellular localization of some wheat histones. featured a $\beta$-sheet (Table 3 Suppl.). The transient expression experiments show that the GFP signal in transformants expressing GFP alone was dispersed throughout the cell (Fig. $3 A$ ), in that produced by the TaH2A-3 and TaH2A-4 fusions GFP accumulated mostly in the nucleus with only a weak signal in the cytoplasm; the signal for the other transformants was restricted to the nucleus (Fig. $3 B$ ).

To primarily confirm their in planta role, these histone variant genes were transformed into $A$. thaliana for ectopic overexpression. At least three independent homozygous transgenic lines of each gene, which were integrated with a one-copy transgene that showed 3:1 kanamycinresistance/-sensitive separation of $\mathrm{T} 2$ generation, were generated (Table 4 Suppl.). The transgenes were amplified in all transgenic lines by a genomic PCR assay (Table 4 Suppl., Fig. 3 Suppl.), showing they were stably integrated into $A$. thaliana genome. The RT-sqPCR analysis showsd that four of the genes (TaH1-1, TaH2A-2, TaH2A-3, and TaH2A.Z-1) were transcribed in the transgenic lines, but the other eight genes were silenced (Table 4 Suppl., Fig. 3 Suppl.).

Two independent homozygous transgenic lines showing a high transgene transcript abundance for each of the four actively transcribed wheat histone variant genes were selected for analyzing the role of wheat histone variants. For seedlings grown on agar plates, neither primary root length nor lateral root density differed between the wild type (non-transgenic) control, plants carrying an empty vector, and the histone gene transgenic lines (Table 5 Suppl.). For soil-grown plants, there was also no discernible phenotype associated with the presence of the transgene (Table 5 Suppl.). With respect to the seedling response to abiotic stresses, exposure to $\mathrm{NaCl}$, mannitol, or $\mathrm{H}_{2} \mathrm{O}_{2}$ significantly restricted germination, whereas the presence of ABA delayed it; however, the transgenic lines behaved identically to the control seedlings (Table 6 Suppl.). Similarly, although seedling growth was significantly inhibited by exposure to $\mathrm{NaCl}$, mannitol, $\mathrm{H}_{2} \mathrm{O}_{2}, \mathrm{ABA}$, NAA, or ACC, there was no effect of any of the transgenes in this respect (Table 6 Suppl.). The experiment in which water was withheld or $\mathrm{NaCl}$ was irrigated also failed to show any advantage of the heterologous transcription of any of the wheat histone variant genes (Table 6 Suppl.).

\section{Discussion}

Like other histones, the 12 wheat histone variants isolated here were rich in lysine and arginine, had high pI, and possessed the $\alpha$-helices necessary for the formation of the histone folding domain (Table 3 Suppl.). Some of the genes had an intron (Table 2 Suppl., Fig. 2 Suppl.), a characteristic that differentiates histone variants from major histones. Unlike major histones, the mRNAs of histone variants are not polyadenylated (Henikoff and Ahmad 2005). In this study, 12 genes were derived from the polyadenylated mRNAs. Based on these, those 12 genes encoded histone variants rather than major histones. The histone genes belong to a class of highly conserved sequences. The 'SR3' genome possesses the whole-genome-scale genetic 
variation (single nucleotide polymorphism as well as insertion and deletion) in comparison with 'JN177'(Wang et al. 2015, Xia 2009), and many isolated abiotic stress responsive genes have allelic variations between 'SR3' and 'JN177' (Li et al. 2010, Liu et al. 2014). However, each of the 12 histone variant genes had no allelic variation between the 2 cultivars (Table 2 Suppl.), indicating histone variant genes are also evolutionarily conserved.

The incorporation of histone variants into chromatin is important for the establishment of an active or inactive transcriptionally poised chromatin structure (Guillemette and Gaudreau 2006). Consistent with other reports (Kumar and Wigge 2010, Smith et al. 2010), the wheat histone variant genes exhibited a diverse transcriptional response to the salinity stress (Fig. 1 and Fig. 1 Suppl.), which implies a relationship between histone variant-regulated chromatin remodeling and the abiotic stress response. The 'SR3' epigenome, at least those aspects related to cytosine methylation, differs substantially from that of its progenitor cultivar 'JN177' (Xia 2009). A DNA methylation regulates gene transcription in concert with histone modification and incorporation of histone variants (Henikoff and Ahmad 2005). Thus, the differential response to $\mathrm{NaCl}$ between 'SR3' and 'JN177' of some histone variant genes may result in the difference in epigenetic regulation of their (salt responsive) targets between the two cultivars, and then contribute to the high salt tolerance ability of 'SR3'.

The heterologous expression of TaH1-1, TaH $2 A-2$, TaH2A-3, and TaH3-1 in A. thaliana had no discernible effect of plant development and response to the abiotic stress (Tables 5 Suppl., 6 Suppl.). Chaperones and other transcription regulation-associated effectors are essential for the recruitment of histone variants to the chromatin (Henikoff and Ahmad 2005). For example, the incorporation of H2A.X to a DNA break site depends on the DNA repair-associated MRE11-RAD50-NBS1 complex, mediator of DNA damage checkpoint protein 1 , and other proteins (West and van Attikum 2006). The replacement of $\mathrm{H} 2 \mathrm{~A}$ by its variant macroH2A to inactivate the $\mathrm{X}$ chromosome depends on the expression of a long non-coding RNA Xist (Okamoto et al. 2004). Thus, although four of the wheat histone variant transgenes were strongly transcribed (Table 4 Suppl., Fig. 3 Suppl.), the necessary interaction with $A$. thaliana intermediaries may not be present to allow their normal incorporation into the chromatin. Most probably this failure was due to differences in the sequences of the wheat histone variants and their $A$. thaliana homologs since alterations in just a few residues can greatly influence the interaction ability of native histones with chaperones (Henikoff and Ahmad 2005, Tachiwana et al. 2008).

An alternative scenario is that the wheat histone variants are readily incorporated into the chromatin by competing with their homologs in A. thaliana, but do not alter either the size or structure of the nucleosomes, so that they do not affect gene expression and phenotype of $A$. thaliana. On the other hand, the incorporation of histone variants has an effect on the compactness of the chromatin - for example, the incorporation of macroH2A makes a more stable nucleosome than does the incorporation of $\mathrm{H} 2 \mathrm{~A}$
(Chakravarthy and Luger 2006), whereas H3.3 and H2A.Z appear to accumulate at active promoters and enhancers (Jin et al. 2009) because they destabilize the nucleosome (Jin and Felsenfeld 2007) and produce less well organized chromatin (Guillemette et al. 2005). The incorporation of histone variants is determined by a well conserved selection mechanism, which ensures the stability and suitability of the chromosome structure (Song et al. 2013). Epigenetic information (such as DNA methylation and histone variant incorporation) reveals a certain plasticity, and it is inherently reversible (Probst et al. 2009). Therefore, there is the possibility that the incorporation of the wheat histone variants compromises the stability and/or suitability of the nucleosome structure; the incorporation is unstable and become weaker in A. thaliana, so that wheat histone variants cannot be incorporated in the transgenic homozygous lines to play roles in abiotic stress response and plant development. The preference of a certain histone variant depends on its interaction with chaperones (Song et al. 2013). Histones can be modified, and indeed some modification is essential to ensure a proper interaction with the chaperones. An intriguing possibility, therefore, is that the wheat histones are modified in some way which blocks their interaction with the chaperones and hence their incorporation into the chromatin in the transgenic homozygous lines, which is an interesting question worthy being studied.

Note that 8 of 12 wheat histone variant genes were not transcribed in $A$. thaliana (Fig. 3 Suppl.) showing that unlike other protein encoding genes, histone variant genes exhibit a high frequency of transgene silencing. Transgene silencing can be brought about in a number of ways (Stewart et al. 2011). A frequently used $35 \mathrm{~S}$ CaMV promoter, for example, can often be inactivated by methylation (Wang et al. 2013). The high frequency of silencing experienced by the wheat histone variant genes may be related to their function, this means that an epigenetic modulation effect of histone variants possibly promotes the epigenetic effect (DNA methylation) of transgene silencing. This provides a way to widen our understanding the mechanism of transgene silencing (the association of transgene silencing with gene function).

In summary, the heterologous expression of four wheat histone variant genes had no effect on the development and abiotic stress response of $A$. thaliana, so for this class of gene, the heterologous expression strategy appears to be an inappropriate means of determining gene function. At the same time, the likelihood is that histone variant genes, although they are involved in the stress response in some way, will not represent suitable candidates for the transgenic improvement of crops.

\section{References}

Chakravarthy, S., Luger, K.: The histone variant macro-H2A preferentially forms "hybrid nucleosomes" - J. biol. Chem. 281: 25522-22531, 2006.

Clough, S.J., Bent, A.F.: Floral dip: a simplified method for Agrobacterium-mediated transformation of Arabidopsis 
thaliana. - Plant J. 16: 735-743, 1998.

Dong, W., Wang, M., Xu, F., Quan, T., Peng, K., Xiao, L., Xia, G.: Wheat oxophytodienoate reductase gene TaOPR1 confers salinity tolerance via enhancement of abscisic acid signaling and reactive oxygen species scavenging. - Plant Physiol. 161: 1217-1228, 2013.

Gao, X., Liu, S.W., Sun, Q., Xia, G.M.: High frequency of HMW-GS sequence variation through somatic hybridization between Agropyron elongatum and common wheat. - Planta 231: 245-250, 2010.

Guillemette, B., Bataille, A.R., Gévry, N., Adam, M., Blanchette, M., Robert, F., Gaudreau, L.: Variant histone H2A. Z is globally localized to the promoters of inactive yeast genes and regulates nucleosome positioning. - PLoS Biol. 3: e384, 2005.

Guillemette, B., Gaudreau, L.: Reuniting the contrasting functions of H2A.Z. - Biochem. cell. Biol. 84: 528-535, 2006.

He, Y., Li, W., Lv, J., Jia, Y., Wang, M., Xia, G.: Ectopic expression of a wheat MYB transcription factor gene, TaMYB73, improves salinity stress tolerance in Arabidopsis thaliana. - J. exp. Bot. 63: 1511-1522, 2012.

Henikoff, S., Ahmad, K.: Assembly of variant histones into chromatin. - Annu. Rev. cell. dev. Biol. 21: 133-153, 2005.

Huang, X., Madan, A.: CAP3: a DNA sequence assembly program. - Genome Res. 9: 868-877, 1999.

Jin, C., Felsenfeld, G.: Nucleosome stability mediated by histone variants H3.3 and H2A.Z. - Genes Dev. 21: 1519-1529, 2007.

Jin, C., Zang, C., Wei, G., Cui, K., Peng, W., Zhao, K., Felsenfeld, G.: H3. 3/H2A. Z double variant-containing nucleosomes mark 'nucleosome-free regions' of active promoters and other regulatory regions. - Natur. Genet. 41: 941-945, 2009.

Kamakaka, R.T., Biggins, S.: Histone variants: deviants? - Genes Dev. 19: 295-316, 2005.

Kumar, S.V., Wigge, P.A.: H2A. Z-containing nucleosomes mediate the thermosensory response in Arabidopsis. - Cell 140: 136-147, 2010.

Li, C., Lv, J., Zhao, X., Ai, X., Zhu, X., Wang, M., Zhao, S., Xia, G.: TaCHP: A Wheat zinc finger protein gene down-regulated by abscisic acid and salinity stress plays a positive role in stress tolerance. - Plant Physiol. 154: 211-221, 2010.

Liu, C., Li, S., Wang, M., Xia, G.M.: A transcriptomic analysis reveals the nature of salinity tolerance of a wheat introgression line. - Plant mol. Biol. 78: 159-169, 2012.

Liu, S., Liu, S., Wang, M., Wei, T., Meng, C., Wang, M., Xia, G.: A Wheat SRO gene enhances seedling growth and abiotic stress resistance via modulating redox homeostasis and maintaining genomic integrity. - Plant Cell 26: 164-180, 2014.

Livak, K.J., Schmittgen, T.D.: Analysis of relative gene expression data using real-time quantitative PCR and the 2- $-\Delta \mathrm{CT}$ method. - Methods 25: 402-408, 2001.

Okamoto, I., Otte, A.P., Allis, C.D., Reinberg, D., Heard, E.: Epigenetic dynamics of imprinted $\mathrm{X}$ inactivation during early mouse development. - Science. 303: 644-649, 2004.

Peng, Z., Wang, M., Li, F., Lv, H., L, C., Xia, G.: A Proteomic study of the response to salinity and drought stress in an introgression strain of bread wheat. - Mol. cell. Proteomics 8: 2676-2686, 2009.

Probst, A.V., Dunleavy, E., Almouzni, G.: Epigenetic inheritance during the cell cycle. - Nat Rev. mol. cell. Biol. 10: 192-206,
2009.

Scippa, G.S., Michele, M.D., Onelli, E., Patrignani, G., Chiatante, D., Bray, E.A.: The histone-like protein H1-S and the response of tomato leaves to water deficit. - J. exp. Bot. 55: 99-109, 2004.

Shan, L., Li, C., Chen, F., Zhao, S., Xia, G.: A Bowman-Birk type protease inhibitor is involved in the tolerance to salt stress in wheat. - Plant Cell Environ. 31: 1128-1137, 2008.

Smith, A.P., Jain, A., Deal, R.B., Nagarajan, V.K., Poling, M.D., Raghothama, K.G., Meagher, R.B.: Histone H2A. Z regulates the expression of several classes of phosphate starvation response genes but not as a transcriptional activator. - Plant Physiol. 152: 217-225, 2010.

Song, Y., Seol, J.-H., Yang, J.-H., Kim, H.-J., Han, J.-W., Youn, H.-D., Cho, E.-J.: Dissecting the roles of the histone chaperones reveals the evolutionary conserved mechanism of transcription-coupled deposition of H3.3 - Nucl. Acids Res. 41: 5199-5209, 2013

Stewart, C.N., Touraev, A., Citovsky, V., Tzfira, T. (ed): Plant Transformation Technologies. - John Wiley \& Sons, New York 2011.

Tachiwana, H., Osakabe, A., Kimura, H., Kurumizaka, H.: Nucleosome formation with the testis-specific histone H3 variant, H3t, by human nucleosome assembly proteins in vitro. - Nucl. Acids Res. 36: 2208-2218, 2008.

Talbert, P.B., Henikoff, S.: Histone variants - ancient wrap artists of the epigenome. - Nat. Rev. mol. cell. Biol. 11: 264-275, 2010.

Von Arnim, A.: Subcellular localization of GUS- and GFP-tagged proteins in onion epidermal cells. - Cold Spring Harbour Protocols: doi:10.1101/pdb.prot4689, 2007.

Wang, M., Liu, C., Xing, T., Wang, Y., Xia, G.: Asymmetric somatic hybridization induces point mutations and indels in wheat. - BMC Genomics 16: 807, 2015.

Wang, M.C., Peng, Z.Y., Li, C.L., Li, F., Liu, C., Xia, G.M.: Proteomic analysis on a high salt tolerance introgression strain of Triticum aestivum/Thinopyrum ponticum. - Proteomics 8: 1470-1489, 2008.

Wang, X., Duan, C.G., Tang, K., Wang, B., Zhang, H., Lei, M., Kun Lu, Mangrauthia, S.K., Wang, P., Zhu, G., Zhao, Y., Zhu, J.K.: RNA-binding protein regulates plant DNA methylation by controlling mRNA processing at the intronic heterochromatin-containing gene IBM1. - Proc nat. Acad. Sci. USA 110: 15467-15472, 2013.

West, A.G., van Attikum, H.: Chromatin at the crossroads. Meeting on signalling to chromatin epigenetics. - EMBO Rep. 7: 1206-1210, 2006.

Xia, G.: Progress of chromosome engineering mediated by asymmetric somatic hybridization. - J. Genet. Genomics 36: 547-556, 2009.

Xia, G.M., Xiang, F.N., Zhou, A.F., Wang, H., Chen, H.M.: Asymmetric somatic hybridization between wheat (Triticum aestivum L.) and Agropyron elongatum (Host) Nevishi. Theor. appl. Genet. 107: 299-305, 2003.

Zhao, Y., Dong, W., Zhang, N., Ai, X., Wang, M., Huang, Z., Xiao, L., Xi, G.: A wheat allene oxide cyclase gene enhances salinity tolerance via jasmonate signaling. - Plant Physiol. 164: 1068-1076, 2014. 